\title{
Photoionization and photoabsorption cross sections for ionospheric calculations
}

\author{
R. S. Stolarsiki and N. P. Johnson \\ Space Physics Research Laboratory, University of Michigan, Ann Arbor 48105, U.S.A.
}

(Received 25 April 1972)

\begin{abstract}
Abatract-Photoionization and photoabsorption cross sections for $\mathrm{N}_{2}, \mathrm{O}_{2}$ and $\mathrm{O}$ are presented in a form useful for calculation of solar EUV absorption and photoelectron production. The cross sections are based mostly on the data presented in the reviews by BoHOEN (1969) and HUTPuaN (1969).
\end{abstract}

\section{INTroduction}

The starting point in all ionospheric calculations must be the interaction of solar ultraviolet radiation with the atmospheric atoms and molecules. This, of course, first requires a knowledge of the solar flux versus wavelength. As of now, the best available data in the EUV region is that of HINTEREGGER (1970). The next most important information for ionospheric caloulations must certainly be the absorption and ionization cross sections which determine how the solar radiation interacts with the atmosphere. This paper presents a proposed set of photo-absorption and photoionization cross sections for $\mathrm{N}_{2}, \mathrm{O}_{2}$ and $\mathrm{O}$ for use in ionospheric calculations.

The cross sections are presented in two forms. First they are presented in tabular form for the wavelength intervals of HnNTEREGGER's (1970) flux table. The absorption cross section values all come from the reviews of ScHоEN (1969) and Huffuman (1969) or are extrapolations of such data. Table 1 shows these results.

The second form of cross section presentation is analytic fits to the smooth portion of the partial photoionization cross section data given in ScHOEN (1969). The expression used to fit the data is

$$
\sigma_{i}(\lambda)=A_{i}\left(1-{ }^{\lambda /} \lambda_{o i}\right)^{m i}\left(e^{\lambda / b_{i}}-1\right)
$$

where $A_{i}, b_{i}$ and $m_{i}$ are free parameters and $\lambda_{o i}$ is usually the ionization threshold of the ith state. Table 2 shows the parameters for all of the states of $\mathrm{N}_{2}, \mathrm{O}_{2}$ and $\mathrm{O}$ which were modeled. Note that $m_{i}$ is always kept integral for ease of integration if this is so desired. These modeled cross sections were used when extrapolations of existing data were necessary.

\section{Photolonization and Absorption Cross Sections}

$\mathrm{N}_{2}$

Figures 1 and 2 show some of the analytic fits to the experimental data for $\mathrm{N}_{2}$. Table 2 gives the parameters for all of the $\mathrm{N}_{2}$ states modeled. Note that the $C^{2} \Sigma_{u}{ }^{+}$ and $D^{2} \pi_{g}$ states were not modeled because the data of SoHOEN (1969) shows no indication of these states. This is probably to be expected because these states involve double electron excitation. Furthermore the potential curves of Grumore (1965) indicate that the $D^{2} \pi_{s}$ state very likely leads to dissociation. Comes and LESSMan (1964) have measured the dissociative ionization cross section for $\mathrm{N}_{2}$ 
Table 1. Cross sections for Hinteregger flux table

\begin{tabular}{|c|c|c|c|c|c|c|}
\hline \multirow{2}{*}{$\begin{array}{l}\text { Desig. } \\
\text { no. }\end{array}$} & \multirow{2}{*}{$\begin{array}{l}\text { Wavelength } \\
(\AA)\end{array}$} & \multicolumn{5}{|c|}{ Cross sections $(\mathrm{Mb})$} \\
\hline & & $\mathbf{N}_{2}$ Abs. & $\mathbf{N}_{2}$ Ion. & $\mathrm{O}_{2} \mathrm{Abs}$ & $\mathrm{O}_{2}$ Ion. & $O$ Abs. and Ion \\
\hline 1 & $1025 \cdot 7$ & $<10^{-3}$ & & 1.58 & 0.98 & \\
\hline 2 & 990 Group & $<10^{-1}$ & & $3 \cdot 2$ & $2 \cdot 9$ & \\
\hline 3 & $977 \cdot 0$ & 0.7 & & $4 \cdot 0$ & $2 \cdot 5$ & \\
\hline 4 & 972.5 & 370.0 & & $32 \cdot 0$ & $25 \cdot 0$ & \\
\hline 5 & $949 \cdot 7$ & $5 \cdot 2$ & & $6 \cdot 3$ & $3 \cdot 7$ & \\
\hline 6 & 944.5 & 0.5 & & 18.0 & $15 \cdot 0$ & \\
\hline 7 & $937 \cdot 8$ & $10 \cdot 0$ & & $5 \cdot 0$ & $3 \cdot 7$ & \\
\hline 8 & $933 \cdot 4$ & $2 \cdot 0$ & & $5 \cdot 6$ & $3 \cdot 8$ & \\
\hline $\mathbf{9}$ & $930 \cdot 7$ & $4 \cdot 8$ & & $26 \cdot 0$ & $17 \cdot 0$ & \\
\hline 10 & $926 \cdot 2$ & $4 \cdot 0$ & & $18 \cdot 5$ & $16 \cdot 7$ & \\
\hline 11 & $1027-911$ & $5 \cdot 7$ & & $4 \cdot 0$ & $2 \cdot 8$ & \\
\hline 12 & $911-890$ & $6 \cdot 0$ & & $7 \cdot 4$ & $3 \cdot 9$ & 0.07 \\
\hline 13 & 904 & $6 \cdot 3$ & & $11 \cdot 0$ & $3 \cdot 9$ & 0.03 \\
\hline 14 & $890-860$ & $8 \cdot 0$ & & $7 \cdot 4$ & $3 \cdot 8$ & 0.7 \\
\hline 15 & $860-830$ & $7 \cdot 5$ & & $9 \cdot 3$ & $4 \cdot 0$ & $1 \cdot 8$ \\
\hline 16 & 835 Group & $13 \cdot 0$ & & $12 \cdot 2$ & $4 \cdot 4$ & $2 \cdot 2$ \\
\hline 17 & $830-800$ & $2 \cdot 2$ & & 26.0 & $9 \cdot 3$ & $2 \cdot 8$ \\
\hline 18 & $911-800$ & $7 \cdot 7$ & & $11 \cdot 0$ & $5 \cdot 0$ & $1 \cdot 4$ \\
\hline 18 & $800-770$ & $27 \cdot 0$ & $11 \cdot 2$ & $25 \cdot 0$ & $12 \cdot 1$ & $\mathbf{3} \cdot \mathbf{6}$ \\
\hline 20 & $790 \cdot 2,790 \cdot 1$ & $25 \cdot 0$ & $11 \cdot 5$ & $28 \cdot 0$ & $10 \cdot 0$ & $3 \cdot 5$ \\
\hline 21 & $787 \cdot 7$ & $9 \cdot 0$ & $8 \cdot 0$ & $24 \cdot 0$ & $13 \cdot 0$ & $3 \cdot 6$ \\
\hline 22 & $786 \cdot 5$ & $9 \cdot 0$ & $8 \cdot 5$ & $24 \cdot 0$ & $11 \cdot 1$ & $3 \cdot 6$ \\
\hline 23 & 780.3 & $19 \cdot 0$ & $9 \cdot 5$ & $28 \cdot 0$ & $11 \cdot 0$ & 3.7 \\
\hline 24 & $770 \cdot 4$ & $15 \cdot 0$ & $14 \cdot 4$ & $18 \cdot 0$ & $11 \cdot 0$ & $3 \cdot 9$ \\
\hline 25 & $770-740$ & $24 \cdot 0$ & $14 \cdot 0$ & $19 \cdot 0$ & $12 \cdot 0$ & $4 \cdot 1$ \\
\hline 26 & $765 \cdot 1$ & $85 \cdot 0$ & $66 \cdot 0$ & $23 \cdot 0$ & $12 \cdot 0$ & $4 \cdot 0$ \\
\hline 27 & 760 & 16.0 & $15 \cdot 9$ & $19 \cdot 0$ & $11 \cdot 0$ & $4 \cdot 1$ \\
\hline 28 & $740-710$ & $18 \cdot 0$ & $13 \cdot 5$ & $31 \cdot 5$ & 27.0 & 4.4 \\
\hline 29 & $710-680$ & $21 \cdot 5$ & $21 \cdot 5$ & $24 \cdot 0$ & $20 \cdot 0$ & $8 \cdot 0$ \\
\hline 30 & 703 Group & $19 \cdot 7$ & $19 \cdot 7$ & $28 \cdot 0$ & $21 \cdot 5$ & $7 \cdot 3$ \\
\hline 31 & $800-630$ & $18 \cdot 0$ & $16 \cdot 7$ & $25 \cdot 0$ & $16 \cdot 8$ & $5 \cdot 9$ \\
\hline 32 & $629 \cdot 7$ & $24 \cdot 0$ & $24 \cdot 0$ & $30 \cdot 0$ & $29 \cdot 0$ & $12 \cdot 3$ \\
\hline 33 & $625 \cdot 3$ & $24 \cdot 0$ & $23 \cdot 0$ & 25.0 & $24 \cdot 0$ & $12 \cdot 4$ \\
\hline 34 & $609 \cdot 8$ & $24 \cdot 0$ & $24 \cdot 0$ & $27 \cdot 0$ & $25 \cdot 0$ & $12 \cdot 6$ \\
\hline 35 & $599 \cdot 6$ & $23 \cdot 0$ & $22 \cdot 0$ & $28 \cdot 0$ & $27 \cdot 0$ & $12 \cdot 6$ \\
\hline 36 & $584 \cdot 3$ & $23 \cdot 0$ & $23 \cdot 0$ & $23 \cdot 0$ & 23.0 & $12 \cdot 4$ \\
\hline 37 & 554 Group & $25 \cdot 0$ & $24 \cdot 0$ & $26 \cdot 0$ & $25 \cdot 0$ & $11 \cdot 6$ \\
\hline 38 & $521 \cdot 0$ & $25 \cdot 0$ & $24 \cdot 0$ & $24 \cdot 5$ & 23.9 & $10 \cdot 6$ \\
\hline 39 & 508 Group & $23 \cdot 0$ & $23 \cdot 0$ & $23 \cdot 0$ & $22 \cdot 5$ & $10 \cdot 2$ \\
\hline 40 & 504 & $26 \cdot 8$ & $26 \cdot 8$ & $23 \cdot 6$ & $23 \cdot 6$ & $10 \cdot 0$ \\
\hline 41 & $499 \cdot 3$ & 26.9 & $26 \cdot 9$ & $23 \cdot 5$ & $23 \cdot 5$ & $9 \cdot 9$ \\
\hline 42 & $465 \cdot 2$ & $24 \cdot 2$ & $24 \cdot 2$ & $21 \cdot 3$ & $21 \cdot 3$ & $9 \cdot 0$ \\
\hline 43 & $630-460$ & $24 \cdot 0$ & $24 \cdot 0$ & $23-0$ & $23 \cdot 0$ & $11 \cdot 0$ \\
\hline 44 & $460-370$ & $19 \cdot 8$ & $19 \cdot 8$ & $18 \cdot 6$ & $18 \cdot 6$ & $8 \cdot 3$ \\
\hline 45 & $368 \cdot 1$ & $16 \cdot 1$ & $16 \cdot 1$ & $15 \cdot 8$ & $15 \cdot 8$ & $8 \cdot 0$ \\
\hline 46 & $364 \cdot 8$ & $15 \cdot 8$ & $15 \cdot 8$ & $15 \cdot 6$ & $15 \cdot 6$ & $8 \cdot 0$ \\
\hline 47 & $360 \cdot 7$ & $15 \cdot 5$ & $15 \cdot 5$ & $15 \cdot 2$ & $15 \cdot 2$ & $8 \cdot 0$ \\
\hline 48 & $335 \cdot 4$ & $13 \cdot 4$ & $13 \cdot 4$ & $13 \cdot 3$ & $13 \cdot 3$ & $7 \cdot 7$ \\
\hline 49 & $303 \cdot 8$ & 12 & 12 & $11 \cdot 0$ & $11 \cdot 0$ & $7 \cdot 3$ \\
\hline 50 & $284 \cdot 1$ & $9 \cdot 8$ & $9 \cdot 8$ & $9 \cdot 7$ & $9 \cdot 7$ & $7 \cdot 1$ \\
\hline
\end{tabular}


Table 1 (cont.)

\begin{tabular}{|c|c|c|c|c|c|c|}
\hline \multirow{2}{*}{$\begin{array}{l}\text { Degig. } \\
\text { no. }\end{array}$} & \multirow{2}{*}{$\begin{array}{c}\text { Wavelength } \\
(\AA)\end{array}$} & \multicolumn{5}{|c|}{ Cross sections $(\mathrm{Mb})$} \\
\hline & & $\mathrm{N}_{2} \mathrm{Abs}$ & $\mathrm{N}_{2}$ Ion. & $\mathrm{O}_{2} \mathrm{Abs}$ & $\mathrm{O}_{2}$ Ion. & O Abs. and ion. \\
\hline 51 & $370-280$ & $12 \cdot 7$ & $12 \cdot 7$ & $12 \cdot 0$ & $12 \cdot 0$ & $7 \cdot 5$ \\
\hline 52 & $280-231$ & $7 \cdot 8$ & $7 \cdot 8$ & $7 \cdot 6$ & $7 \cdot 6$ & $6 \cdot 6$ \\
\hline 53 & 231-205 & $5 \cdot 8$ & $5 \cdot 8$ & $5 \cdot 9$ & $5 \cdot 9$ & $5 \cdot 8$ \\
\hline $\mathbf{5 4}$ & $205-176$ & $4 \cdot 8$ & $4 \cdot 8$ & $4 \cdot 7$ & $4 \cdot 7$ & $5 \cdot 0$ \\
\hline 55 & $176-153$ & $3 \cdot 8$ & $3 \cdot 8$ & $3 \cdot 8$ & $3 \cdot 8$ & $4 \cdot 3$ \\
\hline 56 & $153-100$ & $2 \cdot 6$ & $2 \cdot 6$ & $2 \cdot 7$ & $2 \cdot 7$ & $3 \cdot 3$ \\
\hline 57 & $128-120$ & $2 \cdot 5$ & $2 \cdot 5$ & $2 \cdot 7$ & $2 \cdot 7$ & $3 \cdot 2$ \\
\hline 58 & $120-110$ & $\mathbf{2 \cdot 2}$ & $2 \cdot 2$ & $2 \cdot 4$ & $2 \cdot 4$ & $\mathbf{3} \cdot \mathbf{0}$ \\
\hline 59 & $103 \cdot 6,105 \cdot 2$ & 1.9 & 1.9 & $2 \cdot 1$ & $2 \cdot 1$ & $2 \cdot 7$ \\
\hline 60 & $110-100$ & $2 \cdot 0$ & $2 \cdot 0$ & $2 \cdot 1$ & $2 \cdot 1$ & $2 \cdot 8$ \\
\hline 61 & $94 \cdot 0,96 \cdot 1$ & $1 \cdot 8$ & $1 \cdot 8$ & $1 \cdot 9$ & 1.9 & $2 \cdot 5$ \\
\hline 62 & $100-90$ & $1 \cdot 8$ & $1 \cdot 8$ & $1 \cdot 9$ & 1.9 & $2 \cdot 5$ \\
\hline 63 & $80 \cdot 5,86 \cdot 8$ & $1 \cdot 5$ & 1.5 & $1 \cdot 6$ & 1.6 & $2 \cdot 2$ \\
\hline 64 & $90-80$ & 1.5 & 1.5 & $1 \cdot 6$ & $1 \cdot 6$ & $2 \cdot 2$ \\
\hline 65 & $\mathbf{7 6} \cdot 0$ & $1 \cdot 3$ & $1 \cdot 3$ & $1 \cdot 4$ & $1 \cdot 4$ & $2 \cdot 0$ \\
\hline 66 & $80-70$ & $1 \cdot 3$ & $1 \cdot 3$ & $1 \cdot 4$ & $1 \cdot 4$ & $2 \cdot 0$ \\
\hline 67 & $66 \cdot 3$ & $1 \cdot 1$ & $1 \cdot 1$ & $1 \cdot 2$ & $1 \cdot 2$ & $1 \cdot 8$ \\
\hline 68 & $70-60$ & $1 \cdot 1$ & $1 \cdot 1$ & $1 \cdot 2$ & 1.2 & 1.7 \\
\hline 69 & $50 \cdot 5,50 \cdot 7,55 \cdot 3$ & 0.85 & $0 \cdot 85$ & $0 \cdot 95$ & 0.95 & $1 \cdot 4$ \\
\hline 70 & $60-50$ & 0.90 & 0.90 & $1 \cdot 0$ & 1.0 & 1.5 \\
\hline 71 & $44 \cdot 1$ & 0.65 & $0 \cdot 65$ & 0.80 & 0.80 & $1 \cdot 2$ \\
\hline 72 & $50-40$ & 0.70 & 0.70 & 0.80 & 0.80 & $1 \cdot 2$ \\
\hline 73 & $33 \cdot 6$ & 0.48 & 0.48 & 0.60 & 0.60 & 0.9 \\
\hline
\end{tabular}

and found it extremely small, lending further support to the smallness of the $D^{2} \pi_{g}$ cross section. A cross section for the $E^{2} \Sigma$ state of 10 per cent of the extrapolated total was included, but there is no experimental evidence to either confirm or deny this supposition.

Figure 3 shows the sum of the modeled partial cross sections described above in comparison with the total absorption measurements quoted by HUFFMAN (1969). Figure 4 shows the branching ratios derived from the modeled partial cross sections. These branching ratios are tabulated in Table 3 for the Hinteregger wavelength intervals.

$\mathrm{O}_{2}$

The measured photoionization cross section for the ground state of $\mathrm{O}_{2}+$ shown in Fig. 5 is more complicated than any of the other measured partial cross sections for $\mathrm{N}_{2}$ and $\mathrm{O}_{2}$. It has been necessary to use the sum of two cross sections of the form of equation (1) to fit the $\mathrm{O}_{2}{ }^{+}$ground state. Because of this the threshold wavelength parameter $\lambda_{0}$ is not the actual threshold for this state but is a pseudo-threshold for each of the two terms. Table 2 lists the parameters for the fits to all of the $\mathrm{O}_{2}$ partial cross sections. Figures 6 shows the fit to the data for the sum of the $a^{4} \pi_{u}$ and $A^{2} \pi_{u}$ states which are usually not resolved in experimental data. This lack of resolution is generally not important in ionospherie calculation because the required resolution in a photoelectron production spectrum is usually no greater than that in the laboratory cross section experiments. 
Table 2. Parameters for analytic formula

\begin{tabular}{|c|c|c|c|c|c|}
\hline & te: & $\lambda_{0}$ & $\begin{array}{c}\text { Parameters } \\
A\end{array}$ & $b$ & $m$ \\
\hline $\mathrm{N}_{2}^{+}$ & $X^{2} \Sigma_{g}+$ & 796.0 & $3.56 \times 10^{-15}$ & $18 \cdot 0$ & 2 \\
\hline & & $796 \cdot 0$ & $6.52 \times 10^{-1}$ & $74 \cdot 0$ & 4 \\
\hline & $A^{2} \Pi_{u}^{+}$ & $742 \cdot 7$ & $3.91 \times 10^{-13}$ & $17 \cdot 6$ & 3 \\
\hline & & $742 \cdot 7$ & $3.29 \times 10^{-1}$ & $72 \cdot 6$ & 3 \\
\hline & $B^{2} \Sigma_{u}^{+}$ & $661 \cdot 0$ & $9.88 \times 10^{-12}$ & $21 \cdot 0$ & 1 \\
\hline & & $661 \cdot 0$ & $1.72 \times 10^{-1}$ & $121 \cdot 0$ & 1 \\
\hline & $E^{2} \Sigma_{o}^{+}$ & $355 \cdot 0$ & $1.51 \times 10^{-6}$ & $20 \cdot 0$ & 1 \\
\hline $\mathrm{O}_{2}^{+}$ & $x^{2} \Pi_{g}$ & $1026 \cdot 7$ & $1.94 \times 10^{-2}$ & 121.7 & 1 \\
\hline & & $900 \cdot 0$ & $3.03 \times 10^{-1}$ & $44 \cdot 0$ & 10 \\
\hline & & $850 \cdot 0$ & $3 \cdot 10 \times 10^{-20}$ & $13 \cdot 7$ & 4 \\
\hline & & $750 \cdot 0$ & $4.62 \times 10^{-37}$ & $10 \cdot 0$ & 3 \\
\hline & $a^{4} \Pi_{2 k}$ & $769 \cdot 8$ & $1.17 \times 10^{-16}$ & $16 \cdot 4$ & 2 \\
\hline & & $769 \cdot 8$ & $1.44 \times 10^{-1}$ & $144 \cdot 8$ & 1 \\
\hline & $A^{2} \Pi_{u}$ & $736 \cdot 8$ & $1.07 \times 10^{-16}$ & $16 \cdot 8$ & 1 \\
\hline & & $736 \cdot 8$ & $7.49 \times 10^{-1}$ & $166 \cdot 8$ & 1 \\
\hline & $b^{4} \Sigma_{g}+$ & $682 \cdot 3$ & $1.39 \times 10^{-1}$ & 126.9 & $\frac{1}{3}$ \\
\hline & $B^{2} \Sigma_{g}-$ & $613 \cdot 7$ & $2.01 \times 10^{-10}$ & $17 \cdot 9$ & 3 \\
\hline & & $613 \cdot 7$ & $8.14 \times 10^{-1}$ & $223 \cdot 7$ & 1 \\
\hline & $c^{4} \Sigma_{u}^{-}$ & $505 \cdot 4$ & $2.26 \times 10^{-38}$ & $5 \cdot 2$ & 2 \\
\hline & & $505 \cdot 4$ & $4.12 \times 10^{-3}$ & $42 \cdot 7$ & 2 \\
\hline $\mathrm{O}_{2}^{+}$ & Dissoc. & $670 \cdot 0$ & $3.65 \times 10^{-2}$ & $67 \cdot 5$ & 2 \\
\hline $\mathrm{O}^{+}$ & ${ }^{4} \mathrm{~S}$ & $910 \cdot 0$ & $7.52 \times 10^{-2}$ & 100 & 2 \\
\hline & & $910 \cdot 0$ & $4.52 \times 10^{-1}$ & 50 & 12 \\
\hline & ${ }^{2} \mathrm{D}$ & $725 \cdot 0$ & $8.41 \times 10^{-2}$ & 100 & 1 \\
\hline & & $725 \cdot 0$ & $5.40 \times 10^{-1}$ & 50 & 9 \\
\hline & ${ }^{2} \mathbf{P}$ & $670 \cdot 0$ & $7 \cdot 20 \times 10^{-2}$ & 100 & $\mathbf{l}$ \\
\hline & & $670 \cdot 0$ & $3.09 \times 10^{-1}$ & 50 & 8 \\
\hline & ${ }^{4} \mathrm{P}$ & $430 \cdot 0$ & $2.29 \times 10^{-3}$ & 40 & 2 \\
\hline & ${ }^{2} P$ & $310 \cdot 0$ & $4.07 \times 10^{-2}$ & 60 & I \\
\hline
\end{tabular}

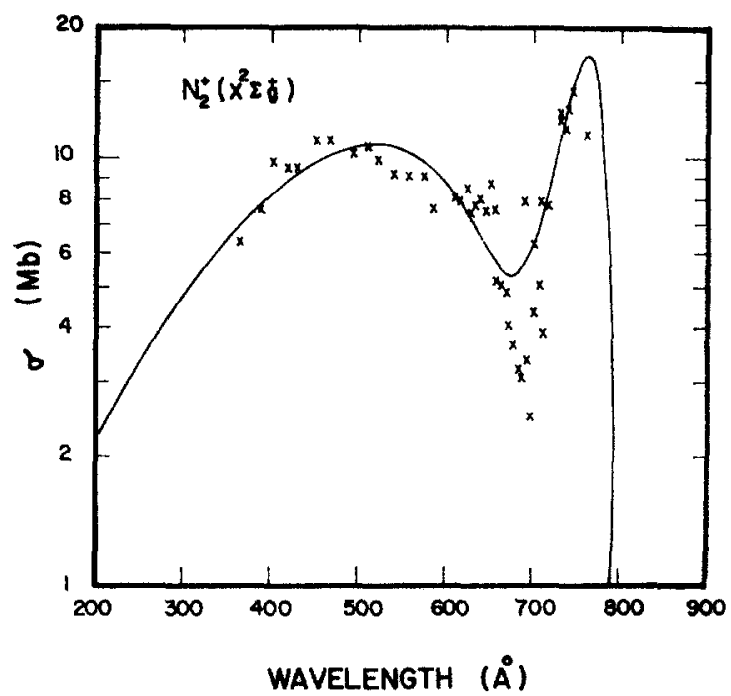

Fig. 1. $\mathrm{N}_{2}{ }^{+}\left(X^{2} \Sigma_{g}{ }^{+}\right)$partial cross section. 


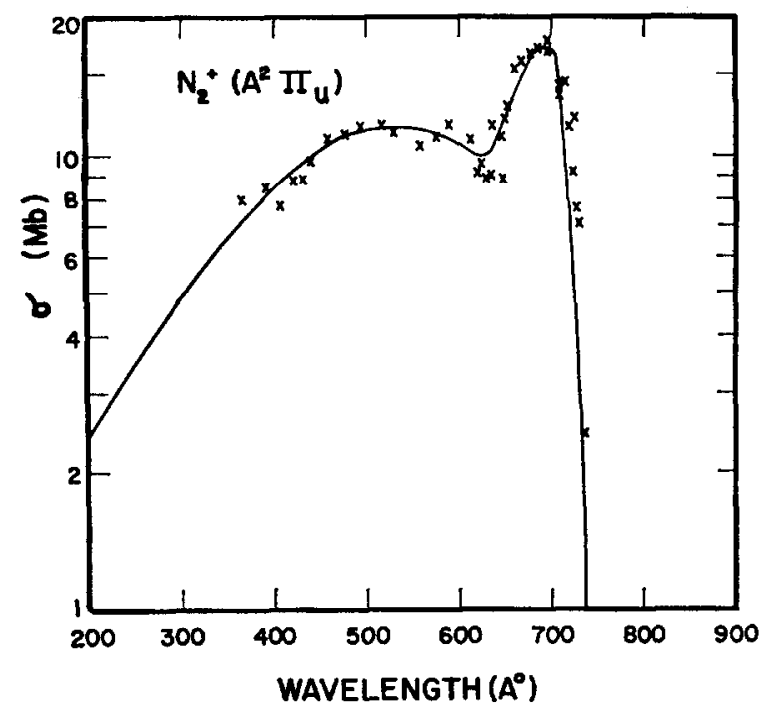

Fig. 2. $\mathrm{N}_{2}+\left(A^{2} \pi_{u}\right)$ partial cross section.

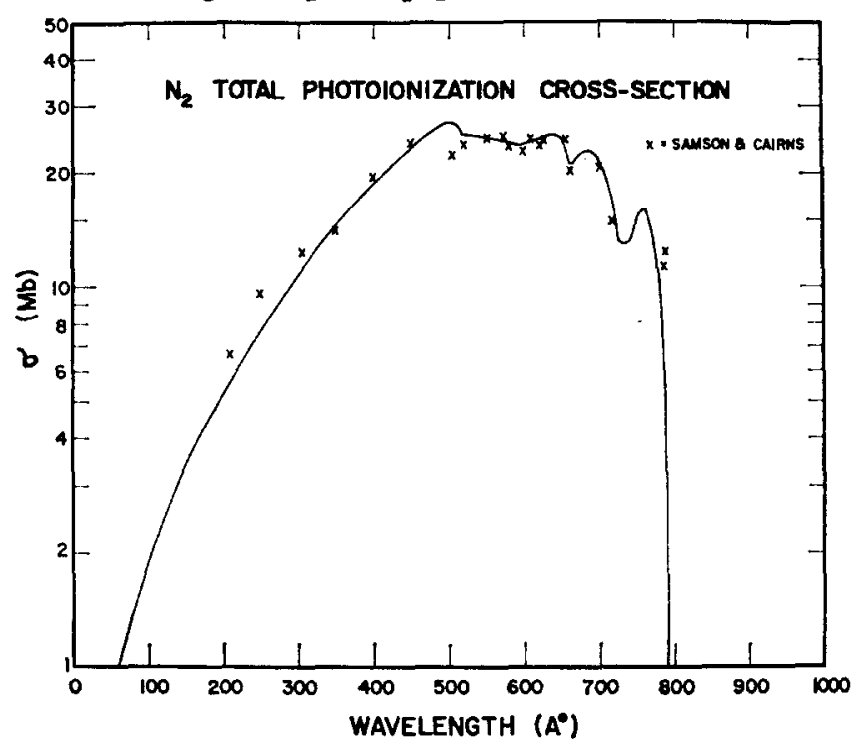

Fig. 3. $\mathbf{N}_{2}$ total photoionization cross section.

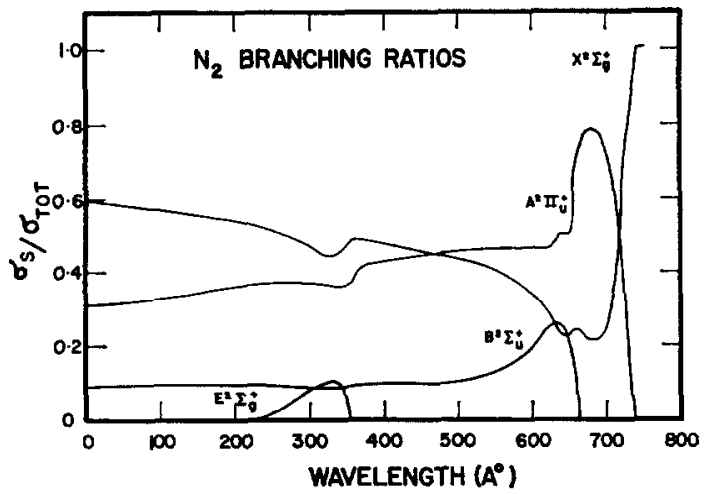

Fig. 4. $\mathrm{N}_{2}$ branching ratios. 
Table 3. $\mathrm{N}_{2}$ and $\mathrm{O}$ branching ratios (first column refers to interval numbers defined in Table 1)

\begin{tabular}{|c|c|c|c|c|c|c|c|c|c|}
\hline \multirow[b]{2}{*}{ Desig. no } & \multirow[b]{2}{*}{$X^{2} \Sigma^{+} g$} & \multicolumn{2}{|c|}{ Nitrogen } & \multirow[b]{2}{*}{$E^{2} \Sigma^{+} g$} & \multirow[b]{2}{*}{${ }^{4} S$} & \multirow[b]{2}{*}{${ }^{2} D$} & \multirow[b]{2}{*}{${ }^{2} P$} & \multirow[b]{2}{*}{${ }^{4} P$} & \multirow[b]{2}{*}{${ }^{2} P$} \\
\hline & & $A^{2} \Pi u$ & $B^{2} \Sigma^{+} u$ & & & & & & \\
\hline 25 & 0.999 & 0.001 & & & $1 \cdot 00$ & 0.0 & 0.0 & $0 \cdot 0$ & $0 \cdot 0$ \\
\hline 28 & 0.68 & 0.32 & & & $1 \cdot 00$ & $0 \cdot 0$ & 0.0 & 0.0 & $0 \cdot 0$ \\
\hline 29 & 0.24 & 0.76 & & & 0.55 & 0.45 & 0.0 & 0.0 & $0 \cdot 0$ \\
\hline 30 & $0 \cdot 26$ & 0.73 & & & $0 \cdot 60$ & $0 \cdot 40$ & 0.0 & 0.0 & $0 \cdot 0$ \\
\hline 31 & 0.56 & $0 \cdot 39$ & 0.05 & & $0 \cdot 75$ & 0.25 & 0.0 & 0.0 & $0 \cdot 0$ \\
\hline 32 & 0.26 & 0.48 & $0 \cdot 26$ & & $0 \cdot 32$ & $0 \cdot 49$ & $0 \cdot 19$ & 0.0 & 0.0 \\
\hline 33 & $0 \cdot 27$ & 0.48 & 0.25 & & $0 \cdot 32$ & 0.49 & $0 \cdot 20$ & $0 \cdot 0$ & $0 \cdot 0$ \\
\hline 34 & 0.31 & 0.47 & 0.22 & & $0 \cdot 30$ & 0.47 & $0 \cdot 23$ & $0 \cdot 0$ & $0 \cdot 0$ \\
\hline 35 & $0 \cdot 34$ & 0.47 & 0.19 & & $0 \cdot 29$ & 0.46 & 0.24 & $0 \cdot 0$ & 0.0 \\
\hline 36 & $0 \cdot 37$ & 0.47 & $0 \cdot 16$ & & 0.29 & $0 \cdot 46$ & $0 \cdot 26$ & $0 \cdot 0$ & $0 \cdot 0$ \\
\hline 37 & 0.40 & 0.47 & $0 \cdot 13$ & & 0.28 & 0.44 & $0 \cdot 27$ & 0.0 & $0 \cdot 0$ \\
\hline 38 & 0.43 & 0.46 & $0 \cdot 11$ & & $0 \cdot 29$ & 0.43 & $0 \cdot 28$ & 0.0 & 0.0 \\
\hline 39 & 0.43 & $0 \cdot 46$ & $0 \cdot 11$ & & $0 \cdot 29$ & 0.42 & $0 \cdot 28$ & 0.0 & $0 \cdot 0$ \\
\hline 40 & $0 \cdot 43$ & $0 \cdot 46$ & 0.11 & & $0 \cdot 30$ & $0 \cdot 42$ & $0 \cdot 28$ & $0 \cdot 0$ & $0 \cdot 0$ \\
\hline 41 & 0.43 & 0.46 & 0.11 & & $0 \cdot 30$ & 0.42 & $0 \cdot 28$ & 0.0 & $0 \cdot 0$ \\
\hline 42 & $0 \cdot 45$ & 0.45 & $0 \cdot 10$ & & 0.31 & 0.41 & $0 \cdot 28$ & 0.0 & $0 \cdot 0$ \\
\hline 43 & $0 \cdot 39$ & $0 \cdot 45$ & $0 \cdot 16$ & & $0 \cdot 29$ & 0.43 & 0.28 & $0 \cdot 0$ & $0 \cdot 0$ \\
\hline 44 & $0 \cdot 47$ & 0.43 & $0 \cdot 10$ & & $0 \cdot 32$ & 0.40 & 0.28 & $0 \cdot 0$ & $0 \cdot 0$ \\
\hline 45 & $0 \cdot 49$ & 0.42 & 0.09 & & 0.30 & $0 \cdot 38$ & 0.26 & 0.06 & $0 \cdot 0$ \\
\hline 46 & 0.49 & $0 \cdot 42$ & 0.09 & & $0 \cdot 30$ & $0 \cdot 38$ & $0 \cdot 26$ & 0.06 & $0 \cdot 0$ \\
\hline 47 & 0.49 & 0.42 & 0.09 & & $0 \cdot 30$ & 0.38 & 0.26 & $0 \cdot 06$ & 0.0 \\
\hline 48 & 0.45 & 0.37 & 0.08 & $0 \cdot 10$ & 0.30 & 0.38 & $0 \cdot 26$ & 0.06 & 0.0 \\
\hline 49 & 0.47 & 0.37 & 0.09 & 0.07 & $0 \cdot 30$ & 0.38 & 0.25 & 0.05 & 0.02 \\
\hline 50 & $0 \cdot 49$ & $0 \cdot 37$ & 0.09 & 0.05 & $0 \cdot 29$ & 0.37 & 0.25 & 0.04 & 0.05 \\
\hline 51 & 0.47 & $0 \cdot 38$ & 0.09 & 0.06 & $0 \cdot 30$ & $0 \cdot 38$ & $0 \cdot 26$ & 0.06 & 0.0 \\
\hline 52 & 0.52 & 0.37 & 0.09 & 0.02 & 0.28 & $0 \cdot 36$ & 0.24 & 0.03 & 0.08 \\
\hline 53 & 0.54 & $0 \cdot 36$ & 0.095 & $0 \cdot 005$ & $0 \cdot 29$ & $0 \cdot 37$ & $0 \cdot 24$ & 0.02 & 0.08 \\
\hline 54 & 0.55 & $0 \cdot 35$ & 0.098 & 0.48 & 0.29 & 0.38 & 0.25 & 0.02 & 0.07 \\
\hline 55 & 0.55 & 0.35 & $0 \cdot 10$ & & $0 \cdot 30$ & $0 \cdot 38$ & 0.25 & 0.01 & 0.06 \\
\hline 56 & 0.56 & $0 \cdot 34$ & $0 \cdot 10$ & & $0 \cdot 30$ & $0 \cdot 39$ & 0.25 & $0 \cdot 01$ & 0.05 \\
\hline 57 & 0.56 & $0 \cdot 34$ & $0 \cdot 10$ & & $0 \cdot 30$ & $0 \cdot 39$ & $0 \cdot 25$ & 0.01 & 0.05 \\
\hline 58 & 0.57 & 0.33 & $0 \cdot 10$ & & $0 \cdot 31$ & $0 \cdot 40$ & 0.25 & $0 \cdot 01$ & 0.05 \\
\hline 59 & 0.57 & 0.33 & $0 \cdot 10$ & & $0 \cdot 31$ & 0.40 & 0.25 & $0 \cdot 01$ & 0.05 \\
\hline 60 & 0.57 & 0.33 & $0 \cdot 10$ & & $0 \cdot 31$ & 0.40 & 0.25 & 0.01 & 0.05 \\
\hline 61 & 0.57 & 0.33 & $0 \cdot 10$ & & $0 \cdot 31$ & 0.40 & 0.25 & 0.00 & 0.04 \\
\hline 62 & 0.57 & $0 \cdot 33$ & 0.10 & & $0 \cdot 31$ & $0 \cdot 40$ & 0.25 & 0.00 & 0.04 \\
\hline 63 & 0.57 & $0 \cdot 33$ & $0 \cdot 10$ & & $0 \cdot 31$ & 0.40 & 0.24 & 0.00 & 0.04 \\
\hline 64 & 0.57 & $0 \cdot 33$ & $0 \cdot 10$ & & $0 \cdot 31$ & 0.40 & 0.25 & 0.00 & 0.04 \\
\hline 65 & 0.58 & 0.32 & $0 \cdot 10$ & & $0 \cdot 32$ & $0 \cdot 40$ & 0.25 & 0.00 & 0.04 \\
\hline 66 & 0.58 & 0.32 & $0 \cdot 10$ & & $0 \cdot 32$ & 0.40 & 0.25 & 0.00 & 0.04 \\
\hline 67 & 0.58 & $0 \cdot 32$ & $0 \cdot 10$ & & $0 \cdot 32$ & $0 \cdot 40$ & $0 \cdot 24$ & 0.00 & 0.04 \\
\hline 68 & 0.58 & 0.32 & 0.10 & & $0 \cdot 32$ & $0 \cdot 40$ & 0.24 & 0.00 & 0.04 \\
\hline 69 & 0.58 & 0.32 & $0 \cdot 10$ & & $0 \cdot 32$ & $0 \cdot 40$ & $0 \cdot 24$ & 0.00 & 0.03 \\
\hline 70 & 0.58 & $0 \cdot 32$ & 0.10 & & $0 \cdot 32$ & 0.40 & 0.24 & 0.00 & 0.03 \\
\hline 71 & 0.58 & $0 \cdot 32$ & $0 \cdot 10$ & & 0.32 & $0 \cdot 40$ & 0.24 & 0.00 & 0.03 \\
\hline 72 & 0.58 & 0.32 & $0 \cdot 10$ & & $0 \cdot 32$ & $0 \cdot 40$ & 0.24 & 0.00 & 0.03 \\
\hline 73 & 0.59 & $0 \cdot 31$ & $0 \cdot 10$ & & $0 \cdot 33$ & $0 \cdot 40$ & $0 \cdot 24$ & 0.00 & 0.03 \\
\hline
\end{tabular}




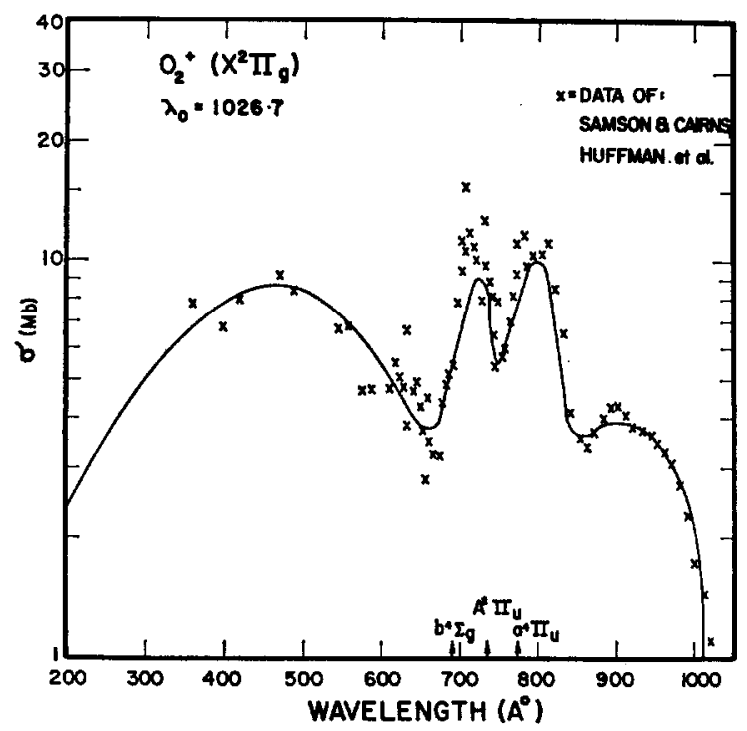

Fig. 5. $\mathrm{O}_{2}+\left(\bar{X}^{2} \pi_{g}\right)$ partial cross section.

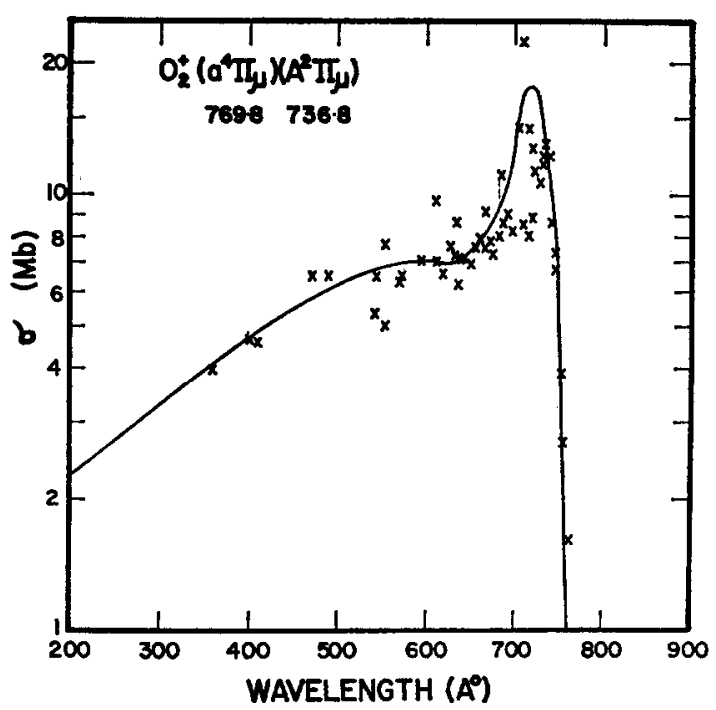

Fig. 6. $\mathrm{O}_{2}+\left(a^{4} \pi_{u}+A^{2} \pi_{u}\right)$ partial cross section.

The total photoionization cross section data is shown in Fig. 7 along with the sum of the modeled partial cross sections. Included in the total is a fit to the dissociative ionization cross section of Comes et al. (1968). The modeled total cross section is slightly higher than the data in just the region where the dissociative cross section is largest, raising the possibility that some of the dissociative ionization is also included in the individual state partial cross sections. Figure 8 and Table 4 give the branching ratios derived from the modeled cross sections. 


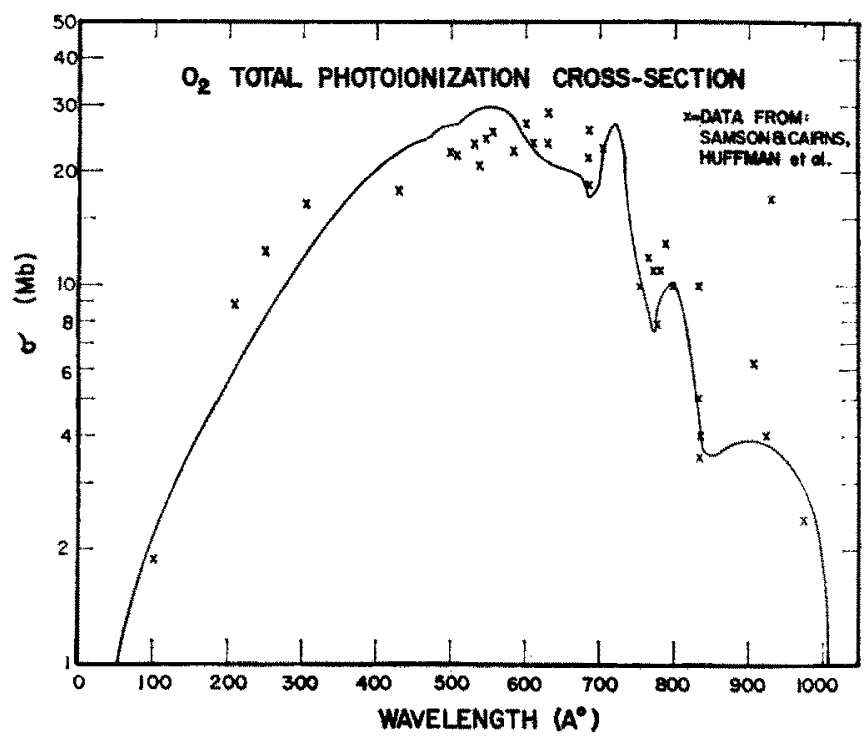

Fig. 7. $\mathrm{O}_{2}$ total photoionization cross section.

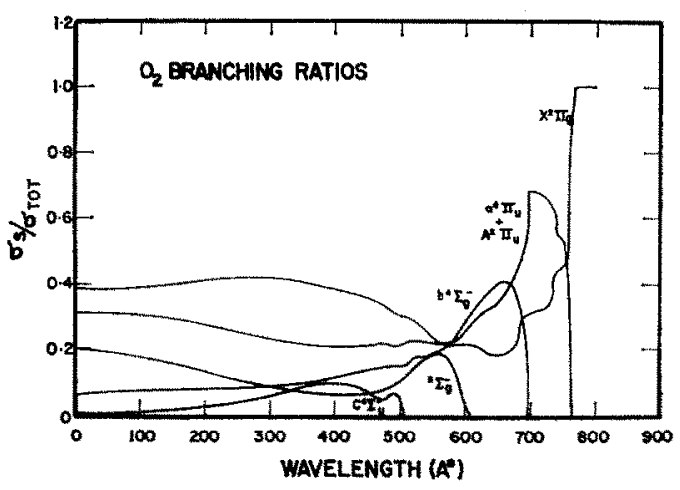

Fig. 8. $\mathrm{O}_{2}$ branching ratios.

SaMsoN (1971) has some data indicating the presence of higher lying states below $300 \AA$. These may improve the fit of the modeled sum of the oross sections to the total ionization measurements.

o

The existing information on atomic oxygen photoionization cross sections is all in the form of total cross sections. Calculations have been made by DaLaARNo et al. (1964) and HuNRY (1967) and measurements by CaIRNS and SAMsoN (1965) and Comes et al. (1968). The magnitudes of the partial cross sections were estimated from the increases at each ionization potential and the shapes assumed are given by the parameters in Table 2. At $\lambda>500 \AA$ the calculations and the data of Comrs 
Table 4. $\mathrm{O}_{2}$ branching ratios (first column refers to interval numbers defined in Table 1)

\begin{tabular}{|c|c|c|c|c|c|c|}
\hline \multirow[b]{2}{*}{ Desig. no. } & \multirow[b]{2}{*}{$x^{2} \Pi g$} & \multicolumn{3}{|c|}{ Molecular oxygen } & \multirow[b]{2}{*}{$c^{\mathbf{c} \Sigma} u$} & \multirow[b]{2}{*}{ Dissoc } \\
\hline & & $\begin{array}{c}+ \\
A^{2} \Pi u\end{array}$ & $b^{4} \Sigma^{-} g$ & $B^{2} \Sigma-g$ & & \\
\hline 25 & 0.65 & 0.35 & & & & \\
\hline 26 & 0.85 & 0.15 & & & & \\
\hline 27 & 0.70 & $0 \cdot 30$ & & & & \\
\hline 28 & 0.36 & 0.64 & & & & \\
\hline 29 & 0.29 & 0.64 & $0 \cdot 07$ & & & \\
\hline 30 & 0.32 & 0.68 & 0.0 & & & \\
\hline 31 & 0.47 & 0.40 & $0 \cdot 12$ & & & 0.01 \\
\hline 32 & 0.20 & 0.33 & 0.40 & & & 0.07 \\
\hline 33 & 0.21 & 0.33 & 0.38 & & & 0.08 \\
\hline 34 & 0.22 & 0.31 & 0.35 & 0.01 & & 0.11 \\
\hline 35 & $0 \cdot 23$ & 0.29 & 0.32 & 0.04 & & 0.12 \\
\hline 36 & 0.22 & $0 \cdot 26$ & 0.26 & $0 \cdot 14$ & & 0.12 \\
\hline 37 & 0.24 & 0.22 & 0.21 & 0.20 & & $0 \cdot 13$ \\
\hline 38 & 0.29 & $0 \cdot 23$ & 0.18 & $0 \cdot 15$ & & $0 \cdot 15$ \\
\hline 39 & 0.31 & 0.23 & 0.18 & 0.13 & & 0.15 \\
\hline 40 & 0.31 & 0.22 & 0.17 & 0.11 & 0.05 & $0 \cdot 14$ \\
\hline 41 & $0 \cdot 31$ & $0 \cdot 22$ & 0.16 & 0.11 & 0.06 & $0 \cdot 14$ \\
\hline 42 & 0.35 & 0.22 & $1 \cdot 15$ & 0.08 & 0.06 & 0.14 \\
\hline 43 & 0.26 & 0.25 & 0.23 & $0 \cdot 11$ & 0.02 & 0.13 \\
\hline 44 & $0 \cdot 38$ & 0.22 & $0 \cdot 12$ & 0.07 & 0.08 & 0.12 \\
\hline 45 & 0.40 & 0.22 & 0.11 & 0.08 & 0.09 & $0 \cdot 10$ \\
\hline 46 & 0.40 & 0.22 & $0 \cdot 11$ & 0.08 & 0.09 & 0.10 \\
\hline 47 & 0.40 & 0.23 & $0 \cdot 10$ & 0.08 & 0.09 & 0.10 \\
\hline 48 & 0.41 & 0.23 & 0.10 & 0.09 & 0.08 & 0.09 \\
\hline 49 & 0.42 & 0.24 & 0.09 & $0 \cdot 10$ & 0.07 & 0.08 \\
\hline 50 & 0.42 & 0.25 & 0.09 & 0.10 & 0.06 & 0.08 \\
\hline 51 & 0.41 & 0.23 & $0 \cdot 10$ & 0.90 & 0.07 & 0.09 \\
\hline 52 & 0.42 & 0.25 & 0.09 & 0.12 & 0.05 & 0.07 \\
\hline 53 & 0.42 & 0.27 & 0.09 & 0.13 & 0.03 & 0.06 \\
\hline 54 & 0.41 & $0 \times 28$ & 0.08 & $0 \cdot 14$ & 0.03 & 0.06 \\
\hline 55 & 0.41 & 0.29 & 0.08 & 0.15 & 0.02 & 0.05 \\
\hline 56 & 0.39 & $0 \cdot 30$ & 0.08 & $0 \cdot 17$ & 0.01 & 0.05 \\
\hline 57 & 0.39 & $0 \cdot 30$ & 0.08 & 0.17 & 0.01 & 0.05 \\
\hline 58 & $0 \cdot 39$ & $0 \cdot 31$ & 0.08 & 0.17 & 0.01 & 0.04 \\
\hline 59 & 0.39 & 0.31 & 0.07 & 0.18 & 0.01 & $0 \cdot 04$ \\
\hline 60 & $0 \cdot 39$ & 0.31 & 0.07 & 0.18 & 0.01 & 0.04 \\
\hline 61 & $0 \cdot 39$ & 0.31 & 0.07 & 0.18 & 0.01 & 0.04 \\
\hline 62 & 0.39 & 0.31 & 0.07 & $0 \cdot 18$ & 0.01 & 0.04 \\
\hline 63 & 0.39 & 0.31 & 0.07 & 0.18 & 0.01 & 0.04 \\
\hline 64 & $0 \cdot 39$ & 0.31 & 0.07 & 0.18 & 0.01 & 0.04 \\
\hline 65 & 0.38 & 0.31 & 0.07 & $0 \cdot 19$ & 0.01 & 0.04 \\
\hline 66 & 0.38 & 0.31 & 0.07 & $0 \cdot 19$ & 0.01 & 0.04 \\
\hline 67 & $0 \cdot 38$ & 0.31 & 0.07 & 0.19 & 0.01 & $0 \cdot 04$ \\
\hline 68 & 0.38 & 0.31 & 0.07 & 0.19 & 0.01 & 0.04 \\
\hline 69 & 0.38 & 0.31 & 0.07 & $0 \cdot 19$ & 0.01 & 0.04 \\
\hline 70 & 0.38 & 0.31 & 0.07 & $0 \cdot 19$ & 0.01 & 0.04 \\
\hline 71 & 0.38 & 0.31 & 0.07 & 0.19 & 0.01 & 0.04 \\
\hline 72 & $0 \cdot 38$ & 0.31 & 0.07 & $0 \cdot 19$ & 0.01 & 0.04 \\
\hline 73 & 0.38 & 0.31 & 0.07 & 0.20 & 0.01 & 0.03 \\
\hline
\end{tabular}


et al. (1968) agree fairly well. At shorter wavelengths they begin to disagree and the assumed total cross section is shown in Fig. 9. The corresponding branching ratios are shown in Fig. 4 and Table 3.



Fig. 9. Atomic oxygen total photoionization cross section.



Fig. 10. O branching ratios.

\section{Discussion}

The cross sections presented above are not new, but they represent an attempt to organize the existing data and present it in a usuable form for those concerned with ionospheric calculations. The data available is very sparse and probably not always reliable judging by some of the disagreements between investigators. It is the needs of the users, which this paper attempts to fulfil, which will point out the areas of laboratory measurement where more and better data are needed. Hopefully, those attempting to use this data in ionospheric calculations will, through their needs for further data, stimulate further laboratory studies. 


\section{REFERENCES}

BhrgowtTz J. and CHUPKa W. A. BeYme K. D. and WeLGE K. H. Blake A. J. and CARver J. W. Buakm A. J. and Carter J. W. Catress R. B. and Samson J. A. R. Comes F. J. and Lessman W. Comes F. J., Elzkr A. and Speirer F. Cook G. R. and Merzater P. H.

Dalgarno A., Henry R. J. W. and STEWART A. L.

GITMORE F. R.

Hall L. A. and Huntermgger H. E. Henrey R. J. W.

HINTEREgGer H. E.

Humbman R. E., Tanaka Y. and LAARRABET J. C.

HUTHMaN R. E.

Huprman R. E., Larrabeie J. C. and Tanaka $Y$.

MarR G. V.

McGowan J. W., Vroom D. A. and Comeaux A. R.

Samson J. A. R. and Catrns R. B.

Samson J. A. R.

SCHOEN R. I.

Tohmatso T., Ogawa T. and Tsuruta $H$.

WAINTAN N., WATKRrR W. C. and WEISSLER G. L.
1969 J. chem. Phys. 51, 2341.

$1969 J$. chem. Phys. 51, 5323.

1965 Phys. Letts 18, 387.

1967 J. chem. Phys. 47, 1038.

1965 Phys. Rev. 189, A1403.

$1964 Z$ Z. Naturforsch. 19a, 65.

1968 Z. Naturforsch. 23a, 114.

1964 J. chem. Phys. 41, 321.

1964 Planet. Space Sci. 12, 235.

1965 J. Quant. Spectrose. Rad. Trans. 5, 369.

$1970 J$. geophys. Res. 75, 6959.

1967 Planet. Space Sci. 15, 1747.

1970 Annls Géophys. 26, 547.

$1963 \quad J$. chem. Phys. 39, 910 (1963).

1969 Can.J. Chem. 47, 1823.

$1967 J$. chem. Phys. 46, 2213.

1967 Photoionization Processes in Gases. Academic Press, New York.

1969 J. chem. Phys. 51, 5626.

$1964 J$ Jeophys. Res. 69, 4683.

1970 Phil. Trans. R. Soc. Lond. A868, 141.

1969 Can. J. Chem. 47, 1855.

1965 Rep. Ionos. Space Res. Japan 19, 482.

1955 Phys. Rev. 98, 542. 